\title{
A Nonlinear Robust Controller Design for Ship Dynamic Positioning Based on $L_{2}$-Gain Disturbance Rejection
}

\author{
Guoqing Xia, Jingjing Xue, Ang Guo, Caiyun Liu, and Xinghua Chen \\ College of Automation, Harbin Engineering University, No. 145 Nantong Street, Nangang District, Harbin City, \\ Heilongjiang Province, China
}

Correspondence should be addressed to Jingjing Xue; xuejingjing@hrbeu.edu.cn

Received 1 August 2016; Accepted 22 September 2016

Academic Editor: Defeng He

Copyright (C) 2016 Guoqing Xia et al. This is an open access article distributed under the Creative Commons Attribution License, which permits unrestricted use, distribution, and reproduction in any medium, provided the original work is properly cited.

In ship motion control process, it is difficult to design ship controller due to the effects of environmental disturbances such as wind, waves, current, and unmodelled dynamics. In order to solve these problems, a nonlinear robust controller based on $L_{2}$-gain disturbance rejection is proposed in this paper. To steer ships to the desired position, an error feedback control law on account of Lyapunov functions is designed. Then, to satisfy the $L_{2}$-gain disturbance rejection, proper parameters are chosen based on the system dissipative property. In order to verify the performance of the proposed controller, the MATLAB simulation results in two situations which are without and with the effects of environmental disturbances are demonstrated.

\section{Introduction}

With the exploration and exploitation of ocean resource and improvement of modern technology, ship Dynamic Positioning (DP) control system is increasingly utilized on floating vessel or platform control systems. DP is defined as follows: "Only depend on the propeller can automatically keep the position of floating structure (maintaining in fixed position or presetting tracked for equipment or ship)" [1]. DP control process has experienced several stages, from Proportion Integration Differentiation (PID) control, advanced output feedback control, to nonlinear adaptive control and hybrid control, especially for hybrid DP control which automatically switched among controller set, improving the performance and maneuverability of DP ships [2].

But in ship motion control process, it is difficult to design ship controller due to effects of environmental disturbances such as wind, waves, current, and unmodelled dynamics. And among control methods of DP system, fuzzy control, backstepping control, neural network control, and hybrid control (see, e.g., [3-10]) are commonly used. Not depending on ship's accurate model, robust fuzzy control by using optimal $H^{\infty}$ control technique on DP ships was designed and had good performance in disturbance rejection and fast response and had good robustness [4]. In order to eliminate the effects of uncertainties, backstepping control method was applied to construct appropriate virtual control law. And neural network control of DP ships was utilized to solve the problem of approximate of uncertainties and unmodelled items [5]. Bateman et al. [6] developed a backstepping method improving ability of control response and disturbance rejecting performance under the low and high gain. And the article [11] designed a recursive backstepping controller to maintain ship in fixed position and the controller had good performance of response and maneuvering motion. Chen et al. [1214] proposed the finite time tracking control method to handle the problem of external disturbances and had good performance. The hybrid control was designed to switch the control situation automatically and had good performance to reject changes of external environment [3]. All of these papers proposed kind of methods to solve the problem of environmental disturbances.

In addition, the key to these designed control methods is to ensure system stabilization. $L_{2}$-gain disturbance rejection is especially used to reduce effects of disturbances by choosing proper control schemes and has good performance and robustness. An optimal $L_{2}$-gain disturbance rejection control for linear system was designed based on LyapunovKrasovskii theories [15]. López-Martínez et al. [16] applied the problem of $L_{2}$ disturbance rejection to a twin-rotor 


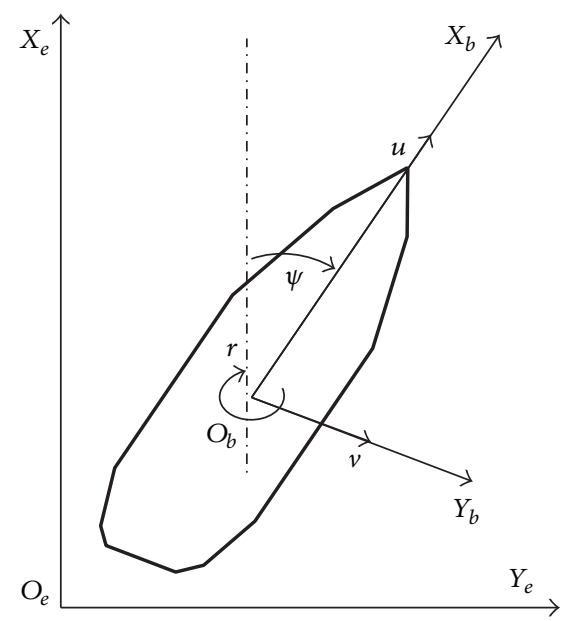

Figure 1: Relationship between Earth-fixed frame and Body-fixed frame.

system and designed the $L_{2}$ control law with PID control to make system stability in variable speed rotors. Paper [17] designed an uncertain switched nonlinear system to solve the problem of $L_{2}$-gain. In order to handle the external disturbance and input constraint, Xiao et al. [18] proposed an adaptive $L_{2}$-gain controller in the condition of attitude tracking in flexible spacecraft. Ahmed [19] proposed an $L_{2}$ gain disturbance rejection neural controller for nonlinear system. Paper [20] designed a disturbance rejection control method in the base of the theories of $L_{2}$-gain and portcontrolled Hamiltonian system. In this paper, based on $L_{2^{-}}$ gain disturbance rejection, the nonlinear robust controller is designed to steer the DP ships to the desired position and solve problems of uncertainties and unmodelled dynamics.

The structure of this paper is organized as follows. Section 2 states the control problem of DP ships subject to uncertain terms and disturbances. Section 3 presents the design of the nonlinear robust controller based on $L_{2}$-gain disturbance rejection. MATLAB simulation results without and with the effects of environmental disturbances are demonstrated to verify the effectiveness of the proposed controller.

\section{Problem Formulation}

2.1. DP Ships' Kinematics and Dynamics. In order to analyze DP ships' kinematics, two reference coordinate systems which include Earth-fixed frame $O_{e}-X_{e} Y_{e} Z_{e}$ and Body-fixed frame $O_{b}-X_{b} Y_{b} Z_{b}$ are introduced in this paper. 3-Degree-OfFreedom (3 DOF) horizontal motion (surge, sway, and yaw) is usually researched in DP control system. And the relationship between Earth-fixed frame and Body-fixed frame is shown as Figure 1.

For the DP control system, the kinematics and dynamics in low speed condition [21] are described as follows:

$$
\begin{aligned}
\dot{\eta} & =R(\psi) \nu, \\
M \dot{v}+D \nu & =\tau_{c}+\Delta,
\end{aligned}
$$

where, $\eta=[x, y, \psi]^{T} \in \mathfrak{R}^{3 \times 1}$ is the vehicle's position vector in Earth-fixed frame. $v=[u, v, r]^{T} \in \mathfrak{R}^{3 \times 1}$ represents the velocities in Body-fixed frame. $M \in \mathfrak{R}^{3 \times 3}$ is the system inertial matrix, $D \in \Re^{3 \times 3}$ represents the strictly positive linear damping matrix, and $\tau_{c} \in \Re^{3 \times 1}$ is the forces and moments vector produced by thrusters. $\Delta \in \mathfrak{R}^{3 \times 1}$ is a vector of forces and moments produced by slowly varying environmental disturbances and unmodelled items. $R(\psi) \in \mathfrak{R}^{3 \times 3}$ is the transformation matrix between Earth-fixed frame and Bodyfixed frame expressed by

$$
R(\psi)=\left[\begin{array}{ccc}
\cos (\psi) & -\sin (\psi) & 0 \\
\sin (\psi) & \cos (\psi) & 0 \\
0 & 0 & 1
\end{array}\right] .
$$

2.2. Control Objective. The DP control system is to steer the ship to maintain a fixed position or preset tracked position. The desired position vector is $\eta_{d}=\left[x_{d}, y_{d}, \psi_{d}\right]^{T}$. The control objective is to design a nonlinear robust control law $\tau$ and choose proper parameters which satisfy the $L_{2}$-gain disturbance rejection constrains. Thus the position $\eta$ is accurately approached to the desired position $\eta_{d}$.

\section{Nonlinear Robust Controller Design Based on $L_{2}$-Gain Disturbance Rejection}

In this section, in order to design a backstepping nonlinear robust controller based on $L_{2}$ disturbance rejection and achieve the control object, the nonlinear robust controller is designed to steer the DP ship to the desired position and solve problems of uncertainties and unmodelled dynamics. The following dynamic equation of DP ship is derived from (1):

$$
M R^{-1}(\psi) \ddot{\eta}+M R_{\operatorname{dot}} \dot{\eta}+D R^{-1}(\psi) \dot{\eta}=\tau_{c}+\Delta .
$$

With defining the error $e \in \mathfrak{R}^{3 \times 1}$ as $e=\eta-\eta_{d}$, the following error dynamic equation based on (3) is written as

$$
\begin{aligned}
M R & (\psi)^{-1} \ddot{e} \\
& +\left(-M R^{-1}(\psi) \dot{R}(\psi) R^{-1}(\psi)+D R^{-1}(\psi)\right) \dot{e} \\
= & M R(\psi)^{-1} \ddot{\eta}_{d}+\tau_{c}+\Delta \\
& +\left(-M R^{-1}(\psi) \dot{R}(\psi) R^{-1}(\psi)+D R^{-1}(\psi)\right) \dot{\eta}_{d} .
\end{aligned}
$$

Simply, let

$$
R_{\mathrm{dot}}=-R^{-1}(\psi) \dot{R}(\psi) R^{-1}(\psi) .
$$

Thus, the error dynamic equation (4) is rewritten as

$$
\begin{aligned}
& M R(\psi)^{-1} \ddot{e}+\left(M R_{\mathrm{dot}}+D R^{-1}(\psi)\right) \dot{e} \\
& \quad=M R(\psi)^{-1} \ddot{\eta}_{d}+\left(M R_{\mathrm{dot}}+D R^{-1}(\psi)\right) \dot{\eta}_{d}+\tau_{c}+\Delta .
\end{aligned}
$$


3.1. $L_{2}$-Gain Disturbance Rejection. $L_{2}$-gain disturbance rejection is used to reduce the effect of disturbances by choosing proper parameters. In order to analyze the $L_{2}$-gain disturbance rejection of a control system, the definitions of $L_{2}$-gain and system dissipation are introduced in following part. And $L_{2}$-gain can be determined by considering the following nonlinear system [15]:

$$
\begin{aligned}
& x=f(x)+g(x) u, \\
& y=h(x),
\end{aligned}
$$

where, $x \in \mathfrak{R}^{n}$ is the state vector of system; $u \in \mathfrak{R}^{p}$ represents the input signal; $y \in \mathfrak{R}^{p}$ is output vector; $f(x), h(x)$, and $g(x)$ represent the given function vector or matrix.

Assumption 1. The system is a free system and has an equilibrium point, that is $f(0)=0$ when $x=0$. At the same time, assume $h(0)=0$; thus for any $u \in L_{2}, y \in L_{2}$, there exists $\gamma \in \mathfrak{R}$ when $L_{2}$-norm $\left\|H_{y u}\right\|$ satisfied the condition of

$$
\left\|H_{y u}\right\|=\sup _{\|u\|_{2} \neq 0} \frac{\|y\|_{2}}{\|u\|_{2}} \leq \gamma .
$$

Therefore, $L_{2}$-norm $\left\|H_{y u}\right\|$ is called $L_{2}$-gain which is less than or equal to $\gamma$. And $\gamma$ is called disturbance rejection factor.

Assumption 2. If a positive semidefinite function $V(x)(V$ : $x \rightarrow R), V(0)=0$, the following inequality is established when

$$
V(x(T))-V(x(0)) \leq \int_{0}^{T} s(u(\tau), y(\tau)) d t
$$

For any $T>0$ and any input signal $u \in R^{p}$, the system is called passive system when inequality (9) is established. And inequality (9) is called dissipative inequality, $s(u, y)$ is energy storage function, and $V(x)$ is the energy storage function.

Consider the following system:

$$
\begin{aligned}
& \dot{x}=f_{1}(x, y), \\
& \dot{y}=f_{2}(x, y)+g_{1}(x, y) u+g_{2}(x, y) w, \\
& z=h(x, y),
\end{aligned}
$$

where $x \in \mathfrak{R}^{n}$ is the state vector of system; $u \in \mathfrak{R}^{p}$ represents input signal; $y \in \mathfrak{R}^{p}$ is output vector; $w \in \mathfrak{R}^{p}$ represents disturbance input signal; $z \in \mathfrak{R}^{p}$ represents evaluation function with characterization of disturbance rejection performance; $f_{1}(*), f_{2}(*), g_{1}(*), g_{2}(*)$, and $h(*)$ are smooth functions with appropriate dimension or matrix. And the dissipative inequality of system (10) is

$$
\dot{V}(x, y) \leq \frac{1}{2}\left(\gamma^{2}\|w\|^{2}-\|z\|^{2}\right)
$$

where $V(x, y)$ is energy storage function with the property of positive semidefinite. Thus the $L_{2}$ disturbance rejection problem is solved by inequality of (11).
3.2. DP Nonlinear Backstepping Controller Design. DP's nonlinear control law is designed based on backstepping theory. The control law $\tau$ is designed by error dynamic equation (6) as

$$
\begin{aligned}
\tau= & -M R(\psi)^{-1} \ddot{\eta}_{e}-\left(M R_{\mathrm{dot}}+D R^{-1}(\psi)\right) \dot{\eta}_{e} \\
& -M R^{-1}(\psi) K \dot{e}-\left(M R_{\mathrm{dot}}+D R^{-1}(\psi)\right) K e+u .
\end{aligned}
$$

Thus (6) can be rewritten as

$$
\begin{aligned}
(\ddot{e}+K \dot{e})= & -M^{-1} R(\psi)\left(M R_{\mathrm{dot}}+D R^{-1}(\psi)\right) \\
& *(\dot{e}+K e)+M^{-1} R(\psi) u+M^{-1} R(\psi) \Delta .
\end{aligned}
$$

In order to design the disturbance rejection control law $u$, define the error varieties $x_{1}, x_{2} \in \mathfrak{R}^{3 \times 1}$ as

$$
\begin{aligned}
& x_{1}=e, \\
& x_{2}=\dot{e}+K x_{1},
\end{aligned}
$$

where $K \in \mathfrak{R}^{3 \times 3}$ is to be designed as parameter matrix; at the same time, let

$$
\begin{aligned}
& F=-M^{-1} R(\psi)\left(M R_{\mathrm{dot}}+D R^{-1}(\psi)\right), \\
& G=M^{-1} R(\psi) .
\end{aligned}
$$

Thus, combining (13), (14), and (15), the derivative of $x_{1}$ and $x_{2}$ is determined as

$$
\begin{aligned}
& \dot{x}_{1}=-K x_{1}+x_{2}, \\
& \dot{x}_{2}=F x_{2}+G u+G \Delta .
\end{aligned}
$$

In order to prove the stability of subsystem $x_{1}$ in (14), Lyapunov function $V_{1}$ is defined as

$$
V_{1}=\frac{1}{2} x_{1}^{T} x_{1}
$$

Thus, the deviation of $V_{1}$ is determined as

$$
\dot{V}_{1}=x_{1}^{T} \dot{x}_{1}=-x_{1}^{T} K x_{1}+x_{1}^{T} x_{2} .
$$

$\dot{V}_{1}=-x_{1}^{T} K x_{1} \leq 0$, when $x_{2}=0$, so the subsystem with $x_{1}$ is stabilization. And to prove the stability of $x_{2}$, Lyapunov function $V_{2}$ is defined as

$$
V_{2}=\frac{1}{2} x_{2}^{T} x_{2}+V_{1}=\frac{1}{2} x_{2}^{T} x_{2}+\frac{1}{2} x_{1}^{T} x_{1} .
$$

Thus deviation of $V_{1}$ is determined as

$$
\dot{V}_{2}=x_{1}^{T} \dot{x}_{1}+x_{2}^{T} \dot{x}_{2}=-x_{1}^{T} K x_{1}+x_{2}^{T}\left(\dot{x}_{2}+x_{1}\right) \text {. }
$$

Combine (16) and (20), so

$$
\dot{V}_{2}=-x_{1}^{T} K x_{1}+x_{2}^{T}\left(F x_{2}+G u+G \Delta+x_{1}\right) \text {. }
$$

Design the feedback control law as

$$
u=G^{-1}\left(-F x_{2}-x_{1}-k x_{2}\right),
$$


where $k>0$ is to be designed as parameter. And plug (22) into (21), so (21) is rewritten as

$$
\dot{V}_{2}=-x_{1}^{T} K x_{1}-k x_{2}^{T} x_{2}+x_{2}^{T} G \Delta .
$$

Thus, if $\Delta=0$

$$
\dot{V}_{2}=-x_{1}^{T} K x_{1}-k x_{2}^{T} x_{2} \leq 0 .
$$

If $\Delta \neq 0$, consider the following inequality:

$$
\begin{aligned}
x_{2}^{T} G \Delta \leq a\left\|x_{2}^{T} G\right\|^{2}+\frac{\|\Delta\|^{2}}{4 a} \leq a\|G\|^{2}\left\|x_{2}\right\|^{2}+\frac{\|\Delta\|^{2}}{4 a} . \\
\text { Let } k=k^{\prime}+a\left\|G^{\prime}\right\| \text {, then } \\
\dot{V}_{2} \leq-x_{1}^{T} K x_{1}-\left(k^{\prime}+a\|G\|^{2}\right) x_{2}^{T} x_{2}+a\|G\|^{2} x_{2}^{2} \\
\quad+\frac{\|\Delta\|^{2}}{4 a} \\
=-x_{1}^{T} K x_{1}-k^{\prime} x_{2}^{2}-a\|G\|^{2} x_{2}^{2}+a\|G\|^{2} x_{2}^{2}+\frac{\|\Delta\|^{2}}{4 a} \\
\leq-x_{1}^{T} K x_{1}-k^{\prime}\left\|x_{2}\right\|^{2}+\frac{\|\Delta\|^{2}}{4 a} .
\end{aligned}
$$

$\dot{V}_{2} \leq 0$ when the following error inequality is workable:

$$
\left\|x_{2}\right\|>\frac{\rho}{2 \sqrt{a k^{\prime}}},
$$

where $\rho$ is the upper bound of $\Delta$. Thus the subsystem with $x_{2}$ is stabilization. Thus the Lyapunov stability can be proved based on the above equations from (13) to (26). And the control law is to be designed to make the system stable under $L_{2}$ disturbance rejection.

3.3. Efficiency Proof of $L_{2}$-Gain Disturbance Rejection. The control law is derived above without considering the capacity of disturbance rejection. And $L_{2}$-gain disturbance rejection of the robust nonlinear controller in this section is proved.

First, evaluation function $z$ mentioned in Section 3.2 is shown as

$$
z=\left[\begin{array}{cc}
h_{1} & 0 \\
0 & h_{2}
\end{array}\right]\left[\begin{array}{l}
x_{1} \\
x_{2}
\end{array}\right],
$$

where $h_{1}, h_{2}$ are the weighted factors. In order to prove the efficiency of $L_{2}$-gain disturbance rejection, the following dissipative inequality needs to be proved:

$$
\dot{V}_{2} \leq \frac{1}{2}\left(\gamma^{2}\|\Delta\|^{2}-\|z\|^{2}\right) .
$$

Define auxiliary function $T$ as

$$
T=2 \dot{V}_{2}-\gamma^{2}\|\Delta\|^{2}+\|z\|^{2} .
$$

Combining (29) with (30), the auxiliary function $T$ is rewritten as

$$
\begin{aligned}
T & =-2 x_{1}^{T} K x_{1}-2 k x_{2}^{T} x_{2}+2 x_{2}^{T} G \Delta-\gamma^{2}\|\Delta\|^{2}+\|z\|^{2} \\
& \leq-x_{1}^{T}\left(K-h_{1}^{T} h_{1}\right) x_{1}-x_{2}^{T}\left(2 k I-h_{2}^{T} h_{2}-\frac{1}{\gamma^{2}} I\right) x_{2} .
\end{aligned}
$$

If the parameters are designed to satisfy the following inequality:

$$
\begin{aligned}
K-h_{1}^{T} h_{1} & \geq 0, \\
2 k I-h_{2}^{T} h_{2}-\frac{1}{\gamma^{2}} I & \geq 0 .
\end{aligned}
$$

Thus $T \leq 0$; that is,

$$
2 \dot{V}_{2}-\gamma^{2}\|\Delta\|^{2}+\|z\|^{2} \leq 0 .
$$

Therefore,

$$
\dot{V}_{2} \leq \frac{1}{2}\left(\gamma^{2}\|\Delta\|^{2}-\|z\|^{2}\right) .
$$

Parameters should satisfy inequality (32); thus the efficiency of $L_{2}$ disturbance rejection can be proved. Therefore the control law is designed as

$$
\begin{aligned}
\tau= & -M R^{-1}(\psi) \\
& \cdot\left(\ddot{\eta}_{e}+(K+k I)\left(\dot{\eta}-\dot{\eta}_{e}\right)+(k K+I)\left(\eta-\eta_{e}\right)\right) \\
- & \left(M R_{\operatorname{dot}}+D R^{-1}(\psi)\right) \\
& \cdot\left(-\left(\dot{\eta}-2 \dot{\eta}_{e}\right)-2 K\left(\eta-\eta_{e}\right)\right) .
\end{aligned}
$$

\section{Simulation Results}

To verify the performance of proposed robust nonlinear controller based on $L_{2}$-gain disturbance rejection, it is assessed in the MATLAB simulation environment with a 3-DOF nonlinear model of DP ship. The desired position vector can be given as follows:

$$
\eta_{d 0}=\left[2,3,5 * \frac{\pi}{180^{\circ}}\right]^{T} .
$$

The initial position and attitude vector is

$$
\eta=[0,0,0]^{T} .
$$

The inertia matrix and damping matrix are given as

$$
\begin{gathered}
M=\left[\begin{array}{ccc}
2.769 e+07 & 0 & 0 \\
0 & 5.160 e+07 & 4.836 e+08 \\
0 & 4.836 e+08 & 4.510 e+10
\end{array}\right], \\
D=\left[\begin{array}{ccc}
8.743 e+04 & 0 & 0 \\
0 & 1.373 e+05 & -3.118 e+06 \\
0 & 2.904 e+06 & 7.264 e+08
\end{array}\right] .
\end{gathered}
$$

The simulating time is $100 \mathrm{~s}$ and the step time is $0.2 \mathrm{~s}$. The desired position is a step signal and its derivate is not to be determined. In order to generate the smoothly desired path, the 2-order filter is applied as

$$
\begin{aligned}
& \dot{\eta}_{d}=\eta_{d}, \\
& \ddot{\eta}_{d}=\omega_{n}^{2} \eta_{d 0}-\omega_{n}^{2} \eta_{d}-\delta\left|\eta_{d}\right| \eta_{d}-2 \zeta \omega_{n} \eta_{d},
\end{aligned}
$$




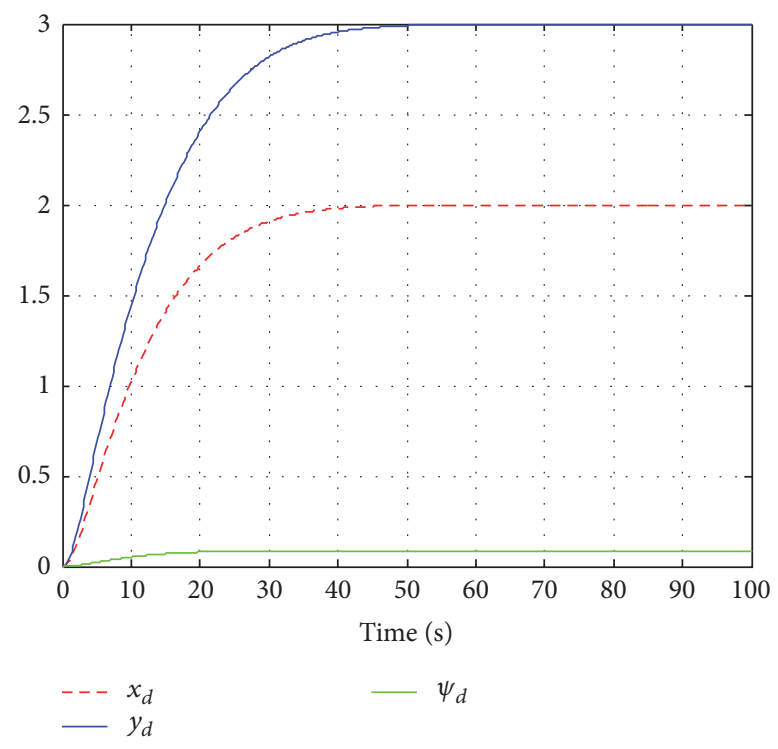

FIGURE 2: The desired position curves.

where $\omega_{n}$ is the natural frequency, $\zeta$ is the relative damping ratio, and $\delta$ is the designed parameter. Thus the desired position $\eta_{d}$ is generated smoothly and the figure of the desired path is shown as Figure 2.

And to validate the effectiveness of controller, two groups of simulating results are demonstrated without and with the effects of environmental disturbances. And let the slowly varying environmental disturbances $\Delta$ be

$$
\begin{aligned}
& \Delta=(\sin (0.02 t)+\cos (0.02 t)) \\
& \text { * diag (1e6 1e6 1e6). }
\end{aligned}
$$

In one situation, without the effects of environmental disturbances, the outputs of ship motion curves (such as the position, position error, and control law $\tau$ ) are shown as Figures 3, 4, and 5. And the parameters in the designed control law are chosen as

$$
\begin{aligned}
K & =\operatorname{diag}\left(\begin{array}{lll}
0.1 & 0.1 & 0.1
\end{array}\right), \\
k & =1.8 .
\end{aligned}
$$

From Figures 3, 4, and 5, the desired path is well approached by the real simulating motion path. And the designed control law $\tau$ effectively steers the ship motion to the desired position though the position error changing in some upper and lower bounds.

The other situation is with the effects of environmental disturbances; the outputs of ship motion curves are demonstrated from Figures 6, 7, and 8. In this condition, the desired path is well approached by the real simulating motion path, and the designed control law $\tau$ effectively controls the ship motion to the desired position although the control law is in shock at the first $10 \mathrm{~s}$. And the position error also can be changed in some acceptable ranges.

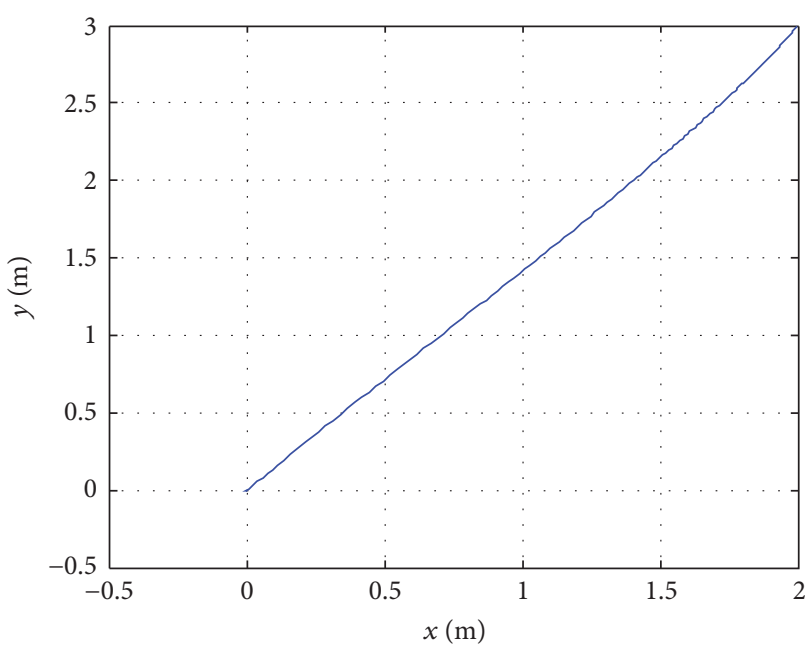

FIGURE 3: Ship horizontal motion without environmental disturbances.
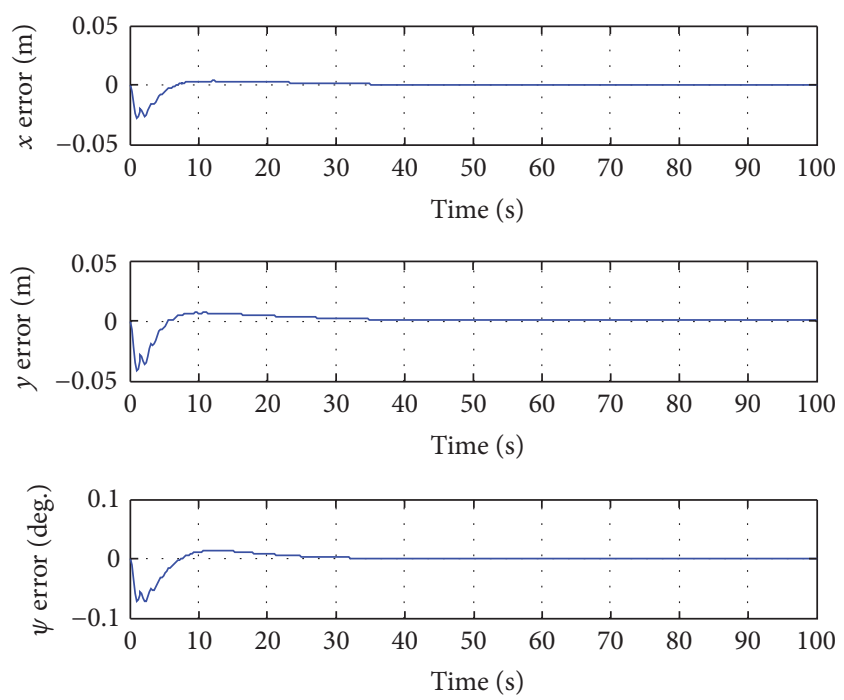

FIGURE 4: The position error of ship without environmental disturbances.
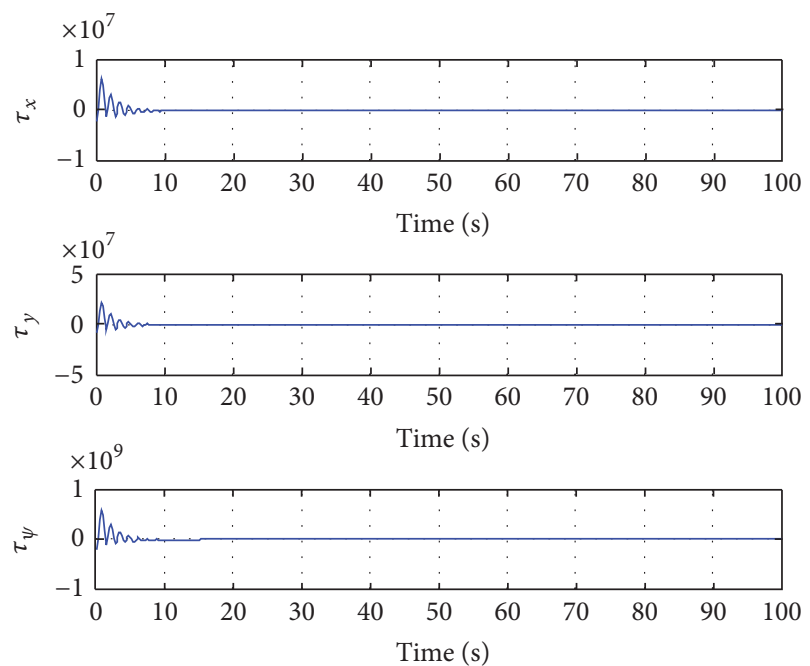

FIgURE 5: The curves of $\tau$ without environmental disturbances. 


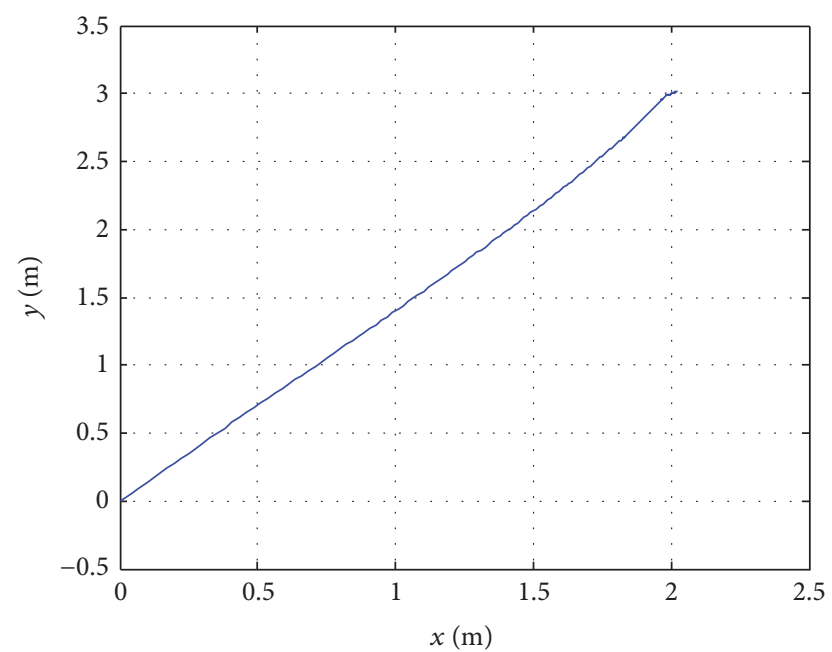

FIGURE 6: Ship horizontal motion with environmental disturbances.
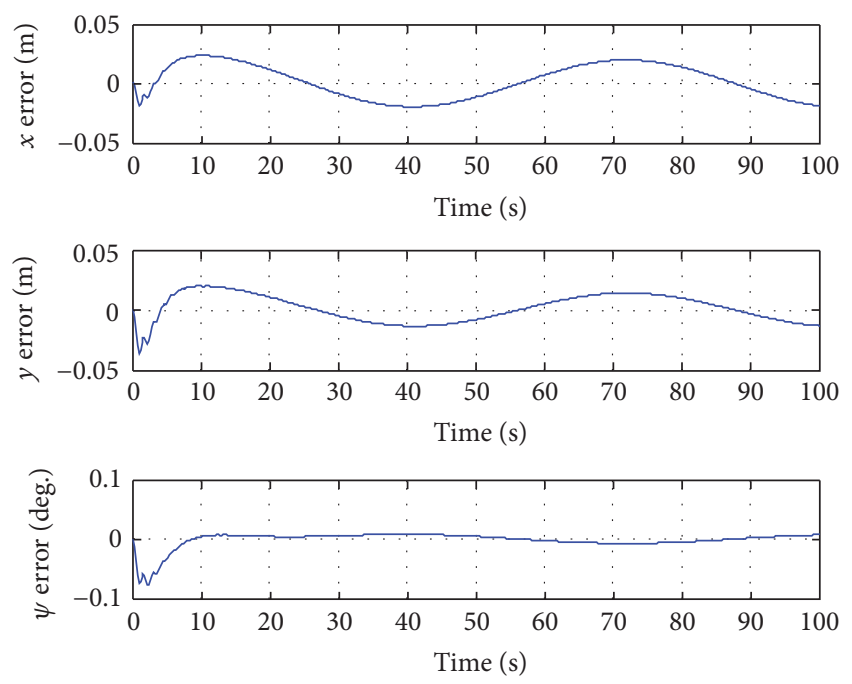

FIGURE 7: The position error of ship with environmental disturbances.
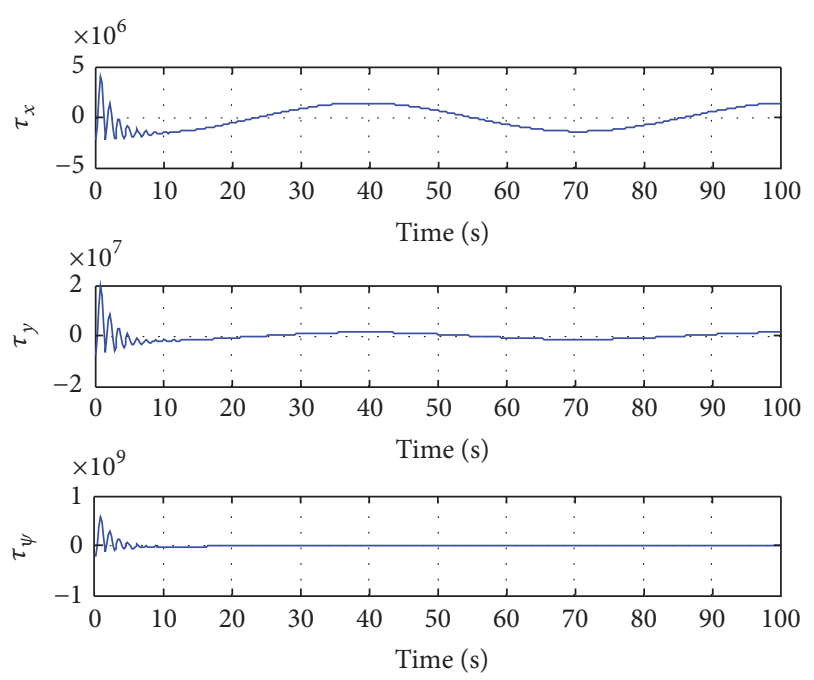

FIGURE 8: The curves of $\tau$ with environmental disturbances.

\section{Conclusions}

In this paper, the problem of Dynamic Positioning for offshore structures has been researched under the nonlinear robust controller based on $L_{2}$-gain disturbance rejection. At first, the nonlinear controller is designed based on robust nonlinear control theories and the efficiency of $L_{2}$ disturbance rejection is proved by designing the parameters. Then, two groups of simulating results are demonstrated without and with the effects of environmental disturbances. And the simulating results validate the performance of the controller.

\section{Competing Interests}

The authors declare that there are no competing interests regarding the publication of this paper.

\section{References}

[1] B. Qian, F. Mingyu, and W. Yuanhui, Ship Dynamic Positioning, Science Press, Beijing, China, 2011.

[2] A. J. Sørensen, "A survey of dynamic positioning control systems," Annual Reviews in Control, vol. 35, no. 1, pp. 123-136, 2011.

[3] T. D. Nguyen, A. J. Sørensen, and S. T. Quek, "Design of hybrid controller for dynamic positioning from calm to extreme sea conditions," Automatica, vol. 43, no. 5, pp. 768-785, 2007.

[4] W. E. Ngongi, J. Du, and R. Wang, "Robust fuzzy controller design for dynamic positioning system of ships," International Journal of Control Automation \& Systems, vol. 14, no. 1, pp. 1-26, 2015.

[5] G. Xia, H. Wu, and X. Shao, "Adaptive filtering backstepping for ships steering control without velocity measurements and with input constraints," Mathematical Problems in Engineering, vol. 2014, Article ID 218585, 9 pages, 2014.

[6] A. Bateman, J. Hull, and Z. Lin, "A backstepping-based low-andhigh gain design for marine vehicles," International Journal of Robust \& Nonlinear Control, vol. 19, no. 4, pp. 480-493, 2009.

[7] G. Xia, C. Pang, and J. Xue, "Fuzzy neural network-based robust adaptive control for dynamic positioning of underwater vehicles with input dead-zone," Journal of Intelligent \& Fuzzy Systems, vol. 29, no. 6, pp. 2585-2595, 2015.

[8] M. D. Thekkedan, C. S. Chin, and W. L. Woo, "Virtual reality simulation of fuzzy-logic control during underwater dynamic positioning," Journal of Marine Science and Application, vol. 14, no. 1, pp. 14-24, 2015.

[9] K. D. Do, "Global robust adaptive path-tracking control of underactuated ships under stochastic disturbances," Ocean Engineering, vol. 111, pp. 267-278, 2016.

[10] T. Elmokadem, M. Zribi, and K. Youcef-Toumi, "Trajectory tracking sliding mode control of underactuated AUVs," Nonlinear Dynamics, vol. 84, no. 2, pp. 1079-1091, 2016.

[11] A. Witkowska, "Control system design for dynamic positioning using vectorial backstepping," Scientific Journals of the Maritime University of Szczecin Zesz, vol. 36, no. 108, pp. 182-187, 2013.

[12] H. Chen, B. Zhang, T. Zhao, T. Wang, and K. Li, "Finite-time tracking control for extended nonholonomic chained-form systems with parametric uncertainty and external disturbance," Journal of Vibration and Control, 2016.

[13] H. Chen, B. Li, B. Zhang et al., "Global finite-time partial stabilization for a class of nonholonomic mobile robots subject 
to input saturation," International Journal of Advanced Robotic Systems, vol. 12, article 159, 2015.

[14] H. Chen, S. Ding, X. Chen, L. Wang, C. Zhu, and W. Chen, "Global finite-time stabilization for nonholonomic mobile robots based on visual servoing," International Journal of Advanced Robotic Systems, vol. 11, article 180, 2014.

[15] P. Millán, L. Orihuela, C. Vivas, and F. R. Rubio, "An optimal control L2-gain disturbance rejection design for networked control systems," in Proceedings of the IEEE American Control Conference (ACC '10), pp. 1344-1349, Baltimore, Md, USA, June 2010.

[16] M. López-Martínez, M. G. Ortega, C. Vivas, and F. R. Rubio, "Nonlinear $\mathrm{L}_{2}$ control of a laboratory helicopter with variable speed rotors," Automatica, vol. 43, no. 4, pp. 655-661, 2007.

[17] M. Wang and J. Zhao, " $\mathrm{L}_{2}$-gain analysis and control synthesis for a class of uncertain switched nonlinear systems," Acta Automatica Sinica, vol. 35, no. 11, pp. 1459-1464, 2009.

[18] B. Xiao, Q.-L. Hu, and G.-F. Ma, "Adaptive L-two-gain controller for flexible spacecraft attitude tracking," Control Theory and Applications, vol. 28, no. 1, pp. 101-107, 2011.

[19] M. S. Ahmed, "Neural controllers for nonlinear state feedback L2-gain control," IEE Proceedings: Control Theory and Applications, vol. 147, no. 3, pp. 239-246, 2000.

[20] X. Liu and Q. Yuan, "Research of permanent magnet linear synchronous motor's disturbance rejection control system of L2 gain based on port-controlled Hamilton," Micromotors, no. 4, pp. 43-48, 2014.

[21] T. I. Fossen, Marine Control Systems, Marine Cybernetics, 2002. 


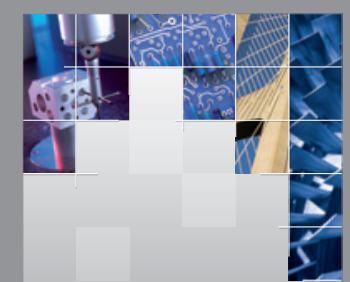

\section{Enfincering}
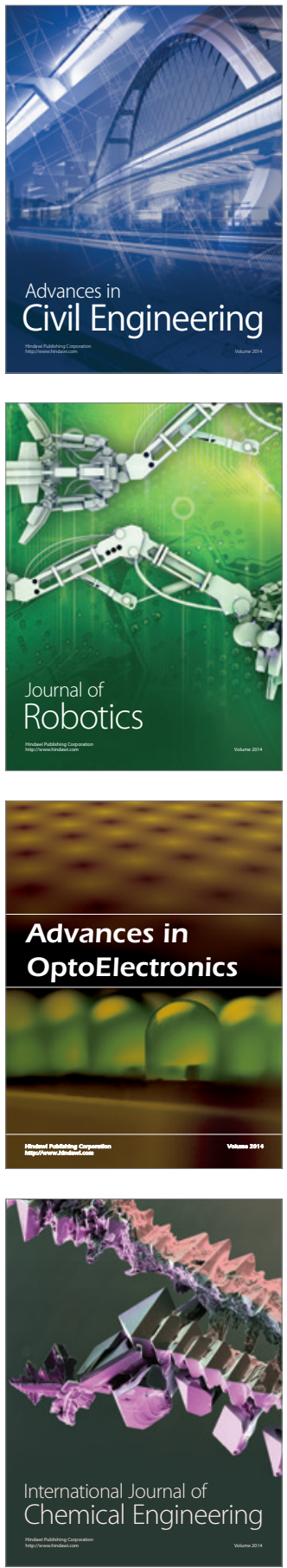

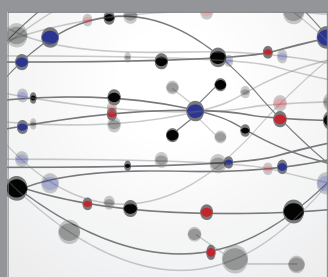

The Scientific World Journal

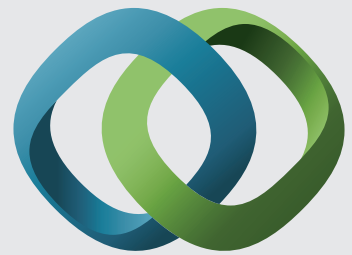

\section{Hindawi}

Submit your manuscripts at

http://www.hindawi.com
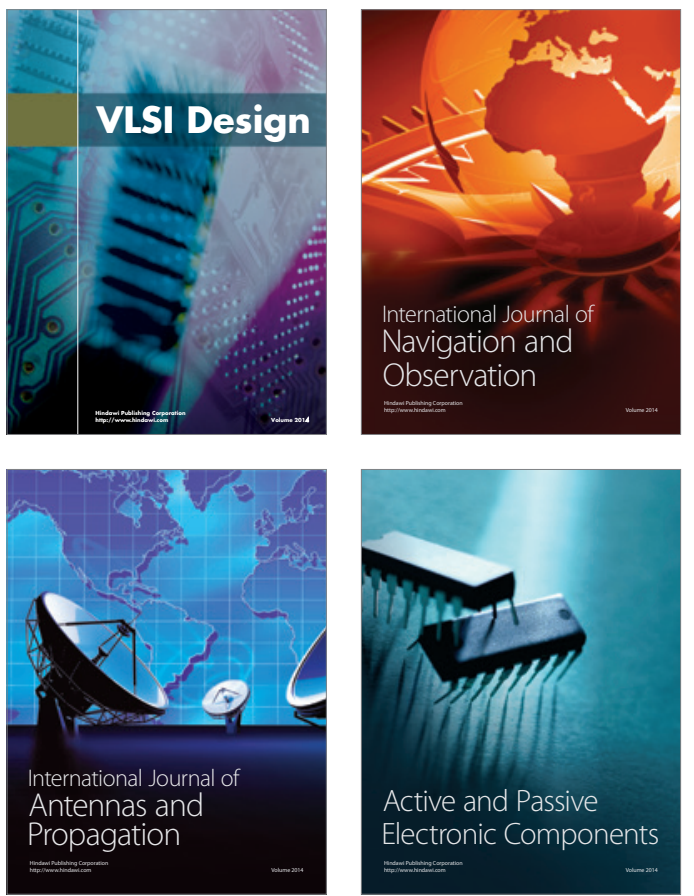
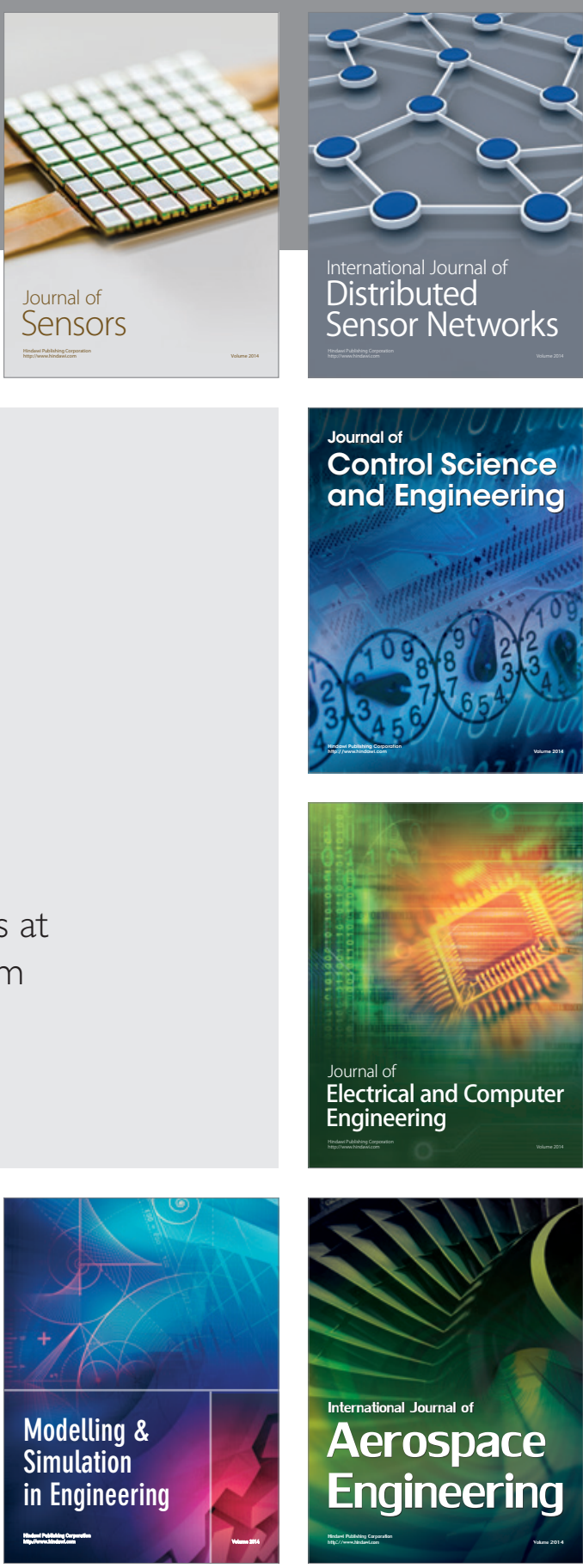

International Journal of

Distributed

Sensor Networks

Journal of

Control Science

and Engineering
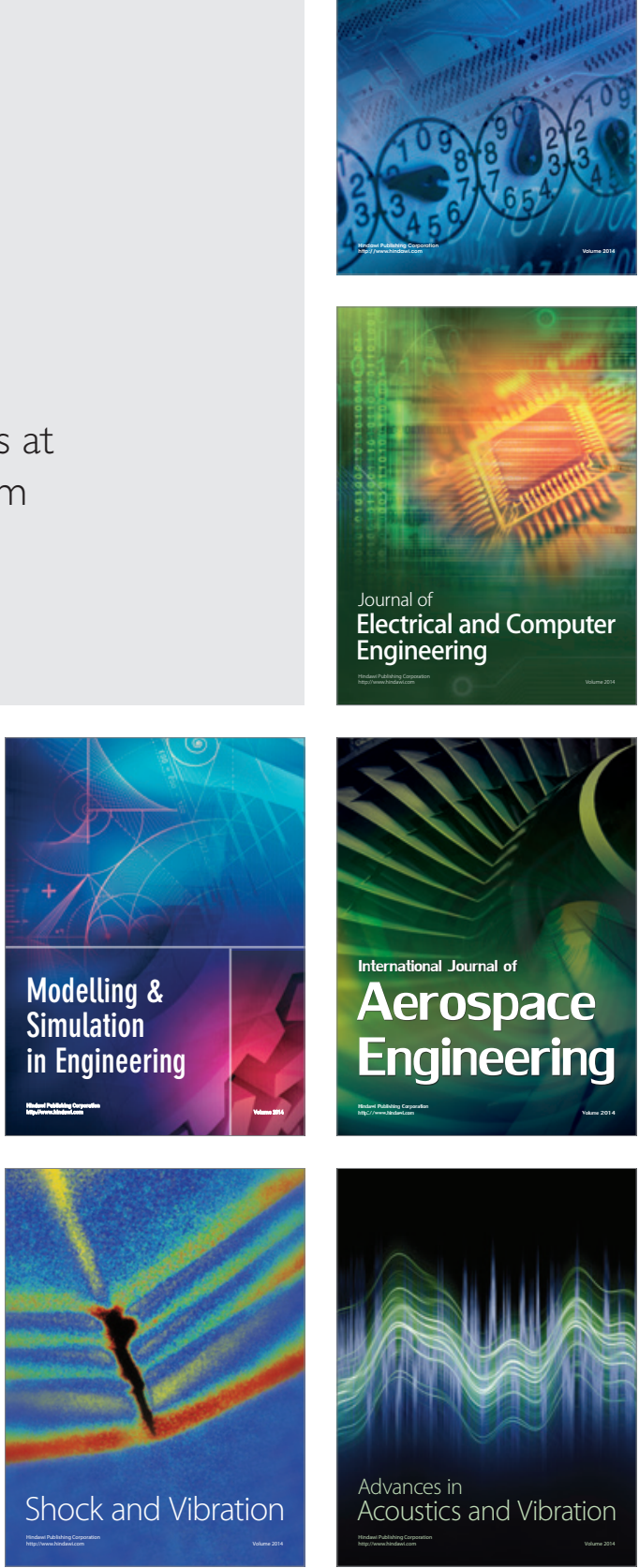